\title{
BNT162b2 mRNA COVID-19 (Comirnaty) Vaccine Effectiveness in Elderly Patients Who Live in Long-Term Care Facilities: A Nationwide Cohort
}

\author{
Sivan Goldin ${ }^{\mathrm{a}, \mathrm{b}}$ Limor Adler ${ }^{\mathrm{b}, \mathrm{c}}$ Joseph Azuri ${ }^{\mathrm{b}, \mathrm{c}}$ Liran Mendel ${ }^{\mathrm{d}}$ Shay Haviv ${ }^{\mathrm{e}}$ Nimrod Maimon ${ }^{\mathrm{f}, \mathrm{g}, \mathrm{h}}$ \\ aSiaal Research Center for Family Medicine and Primary Care, Faculty of Health Sciences, Ben-Gurion University of the \\ Negev, Beer-Sheva, Israel; 'bepartment of Family Medicine, Maccabi Healthcare Services, Tel Aviv, Israel; 'Department

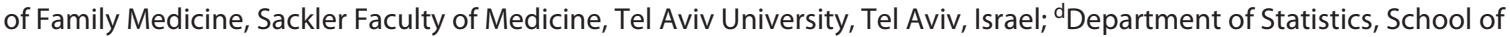 \\ Mathematics, Tel Aviv University, Tel Aviv, Israel; eDepartment of Industry and Management, Aman Consultant, Tel Aviv, \\ Israel; 'Soroka University Medical Center, Faculty of Health Sciences, Ben-Gurion University of the Negev, Beer-Sheva, Israel; \\ gDepartment of Internal Medicine B, Soroka University Medical Center, Faculty of Health Sciences, Ben-Gurion University of \\ the Negev, Beer-Sheva, Israel; 'Israel Ministry of Health, Senior Shield Project, Jerusalem, Israel
}

\section{Keywords}

COVID-19 · Elderly people · Nursing homes · Prevention · Vaccines

\begin{abstract}
Introduction: In early 2020, the novel SARS-CoV-2 virus began to spread around the world and claim victims. Initially, in the Western world, COVID-19-related mortality was due to illness in long-term care facilities (LTCFs). To manage the COVID-19 crisis in LTCFs in Israel, the Ministry of Health established a task force named "Senior Shield." The task force executed a screening program of weekly polymerase chain reaction (PCR) SARS-CoV-2 tests for LTCF residents and caregivers, and at a later stage, the task force led the Ministry of Health vaccination program at LTCFs. This study aimed to estimate the effectiveness of the BNT162b2 mRNA COVID-19 (Comirnaty) vaccine in reducing COVID-19 morbidity and mortality in LTCF residents. Methods: We designed a nationwide cohort study utilizing data from the Senior Shield task force. Residents had received the vaccines starting December 2020. The study follow-up period was 5 months (ending May 2021). We defined four outcomes: (a) documented SARS-CoV-2 infection, defined by a positive PCR test, (b) CO-
\end{abstract}

karger@karger.com www.karger.com/ger

Karger"

BOPEN ACCESS
(C) 2022 The Author(s)

Published by S. Karger AG, Basel

This is an Open Access article licensed under the Creative Commons Attribution-NonCommercial-4.0 International License (CC BY-NC) (http://www.karger.com/Services/OpenAccessLicense), applicable to the online version of the article only. Usage and distribution for commercial purposes requires written permission.
VID-19 death, defined by a positive PCR test followed by death, (c) all-cause mortality, defined as death regardless of the result of a PCR test, and (d) a composite endpoint which included documented SARS-CoV-2 infection or death, the earliest of both. We used Kaplan-Meier curves with a logrank comparison and Cox regression with a time-dependent covariate model to estimate adjusted hazard ratios for vaccine effectiveness (VE). The index date was the date of the first vaccine dose. In unvaccinated residents, the index date was the first date of vaccination in their LTCF. Results: A total of 43,596 residents with a mean age of 83 years living in 454 LTCFs were found eligible for this study. Ninety-one percent of the study population received the first vaccine dose $(39,482)$ and $86 \%$ received the second vaccine dose $(37,656)$. Estimated VE 28 days after the first vaccine dose (approximately 7 days after the second vaccine dose) was $81.2 \%$ for SARS-CoV-2 infection, $85.3 \%$ for COVID-related death, $63.7 \%$ for all-cause mortality, and $71.1 \%$ for the composite endpoint (SARS-CoV-2 infection or death). Conclusion: This study shows that the BNT162b2 mRNA COVID-19 vaccine effectively prevents SARS-CoV-2 infection, COVID-19-related

Sivan Goldin and Limor Adler share equal contribution.
Correspondence to:

Limor Adler, adler_l@mac.org.il 
death, and all-cause mortality in LTCF residents. Further research is warranted on the effect of the third vaccine (booster) in this population.

(C) 2022 The Author(s)

Published by S. Karger AG, Basel

\section{Introduction}

In early 2020, the novel SARS-CoV-2 virus began to spread around the world and claim victims. In the Western world, COVID-19-related mortality occurred primarily in long-term care facilities (LTCFs) [1,2].

Elderly residents of LTCFs tend to have multiple comorbidities, which places them at increased risk of COVID-19 infection [3]. In addition, high prevalence of cognitive impairment also increases the risk of infection [4]. Two additional risk factors for SARS-CoV-2 infection in LTCFs are the crowdedness of residents in these type of facilities and a long-standing shortage of trained personnel [5]. These issues make LTCFs particularly vulnerable to fatal COVID-19 outbreaks. In the USA, it was estimated that LTCF residents represented $42 \%$ of the total number of COVID deaths [6], with fatality rates reaching up to one-third of residents [7].

In the first wave of the COVID-19 outbreak in Israel, residents of LTCFs accounted for 52.4\% of all COVID19-related deaths in the country, despite being approximately $1 \%$ of the country's population [8]. This triggered the Israeli government's concern regarding the elderly population, specifically to their potential burden on the hospitals' medical care abilities, up to the point of insufficiency of the entire Israeli health system. This burden could then impact the whole population, as medical services for routine ailments will become less accessible.

To improve the country's ability to deal with such a catastrophic scenario, and in line with the European Center for Disease Prevention and Control (ECDC) [9] and the Center for Disease Control and Prevention in the USA (CDC) [10], the government decided to establish a dedicated task force to protect roughly 123,000 caregivers and residents of LTCFs against the COVID-19 epidemic [11]. This task force was named "Senior Shield" and was led by experienced medical personnel. Starting July 2020, the task force implemented weekly polymerase chain reaction (PCR) SARS-CoV-2 testing for all residents and caregivers [12]. Following the systematic weekly screening approach, LTCF residents' mortality and morbidity decreased during the second wave of infection, starting in August 2020 [12].

In the first half of 2021, large-scale campaigns to administer COVID-19 vaccines to the population began worldwide, with Israel at the forefront [13-15]. Clinical studies conducted to test the efficacy of the mRNA-based vaccine showed disease prevention at approximately $95 \%$ [16]. In spite of this reassuring rate, very limited data were available about the vaccines' effectiveness on the geriatric population, as this population was not included in the BNT162b2 vaccine clinical trials [17]. Studies conducted later found that the vaccines' immunogenicity in the elderly population was lower than the general population, yet a certain level of protection was acquired [18-21]. Starting December 2020, the Senior Shield task force began vaccinating the residents and caregivers of LTCFs in Israel. This study aimed to estimate the effectiveness of the BNT162b2 vaccine given to residents of LTCFs in Israel in reducing COVID-19 morbidity and mortality.

\section{Materials and Methods}

\section{Study Design and Setting}

We designed a nationwide cohort study using data from the Senior Shield task force. All LTCF residents aged 65 years or older were included in the study. Residents began receiving the vaccines in December 2020. The study follow-up period ended in May 2021 (a total of 5 months). Residents who were diagnosed with a SARSCoV-2 infection prior to receiving the first vaccine dose were excluded from the analysis. The BNT162b2 vaccine was given according to the manufacturer's recommendations in two doses 21 days apart. The Local Ethics Committee (IRB) of the Soroka Medical Center approved this study (0429-20-SOR).

We recorded the dates of positive PCR tests if the resident had documented SARS-CoV-2 infection and the date of death if the resident died during the study and follow-up period. COVID19-related death was defined as cases in which death occurred following a positive result on a SARS-CoV-2 PCR test, and the hospitalizing institute gave a report to the Israeli Ministry of Health, via official death certificate, that the cause of death was a COVID-19 infection. The index date for each vaccinated subject was the date of the first dose. In unvaccinated residents, the index date was the first date of vaccination provided to the other residents in their LTCF. The endpoint was defined as death, SARS-CoV-2 infection, or the last day of the follow-up period (May 19, 2021).

\section{Study Outcomes}

Four outcomes were defined: (a) documented SARS-CoV-2 infection, defined by a positive PCR test, (b) COVID-19-related death, defined by a positive PCR test prior to the death of the patient, (c) all-cause mortality, defined as death regardless of the result of a PCR test, and (d) a composite endpoint which included documented SARS-CoV-2 infection or death, the earliest of both.

\section{Statistical Analysis}

We measured the time from the index date to SARS-Cov-2 infection, COVID-19 death, all-cause mortality, and the composite endpoint using Kaplan-Meier curves with a log-rank comparison. We used a cumulative incidence plot. Risk differences between the two groups were measured at the end of the follow-up. We used a Cox regression with a time-dependent covariate model to estimate 


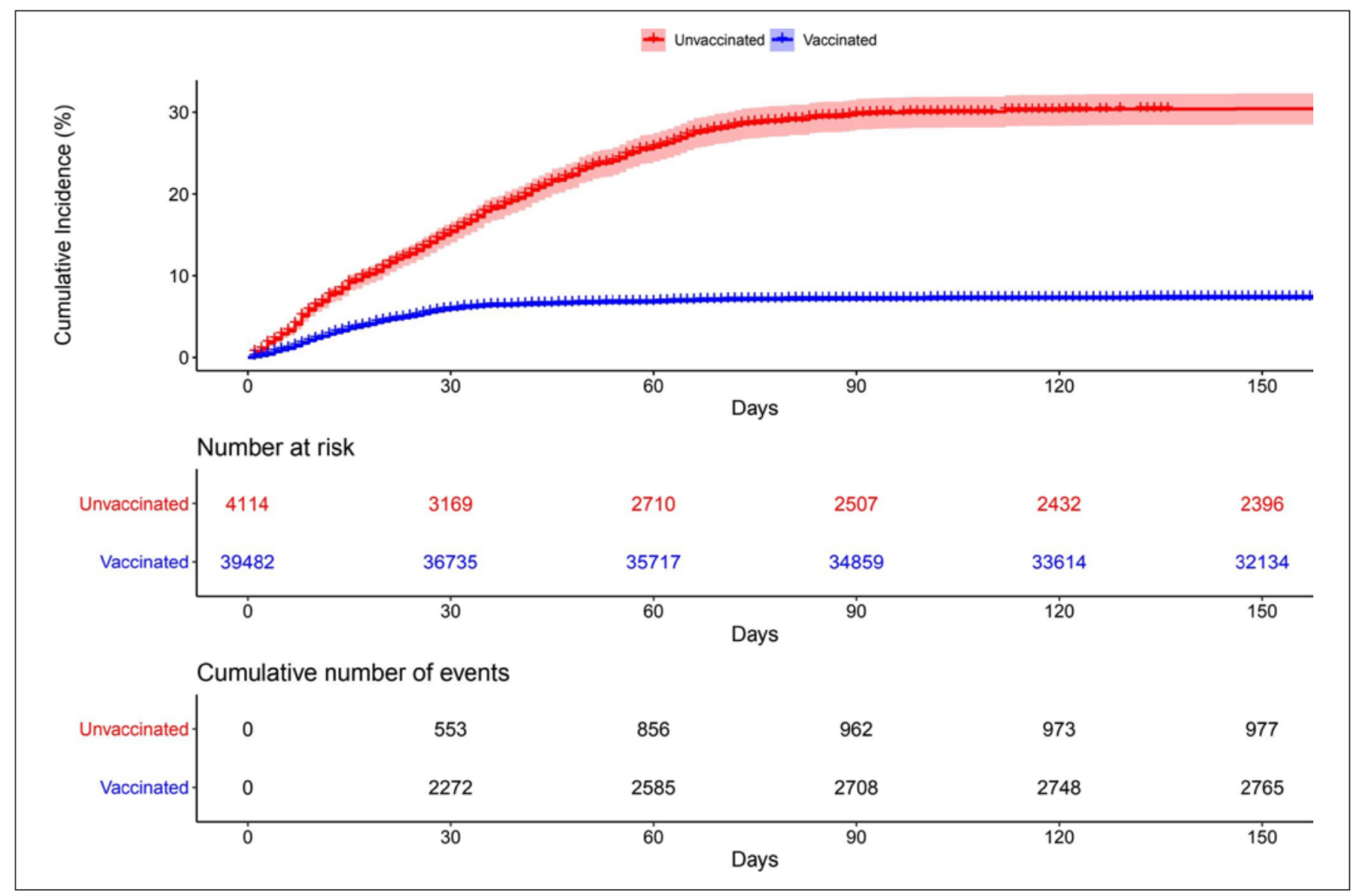

Fig. 1. Documented SARS-CoV-2 infection.

Table 1. Residents' events during the study follow-up period

\begin{tabular}{lll}
\hline & $\begin{array}{l}\text { Vaccinated residents, } \\
39,482(90), n(\%)\end{array}$ & $\begin{array}{l}\text { Unvaccinated residents, } \\
4,14(10), n(\%)\end{array}$ \\
\hline $\begin{array}{l}\text { SARS-CoV-2 infection } \\
\text { COVID-19-related death }\end{array}$ & $2,765(7)$ & $977(23.7)$ \\
$\begin{array}{l}\text { All-cause mortality } \\
\begin{array}{l}\text { Composite endpoint (documented SARS-CoV-2 infection or } \\
\quad \text { death, the earliest of both) }\end{array}\end{array}$ & $\begin{array}{l}2,673(1.4) \\
\text { (6.6) }\end{array}$ & $1,067(25.9)$ \\
\hline
\end{tabular}

adjusted hazard ratios for vaccine effectiveness (VE), 10 days after the first dose and 7 days after second dose, controlling for age and stratified by institution. These periods were defined due to the vaccine effectiveness evident approximately 10 days after the first dose, with maximal response 7 days after the second dose (which is given 21 days after the first dose) [16].

We performed an age-dependent analysis primarily by monitoring if the effect of age was linear, and then used the age variable as a continuous variable with increments of 5 years. For the age-dependent analysis, we removed all patients aged 105 or old- er since results revealed outcomes not linear with all other age groups. This may have occurred due to incorrect age documentation of part of the patients when they immigrated to Israel without any official document at the beginning of the last century $[22,23]$.

The effectiveness was calculated as 1 minus age-adjusted hazard ratio. We used the Statistical Package for Social Sciences (SPSS) software version 27 for data analysis and $\mathrm{R}$ studio version 1.4.1106 to create the figures. 


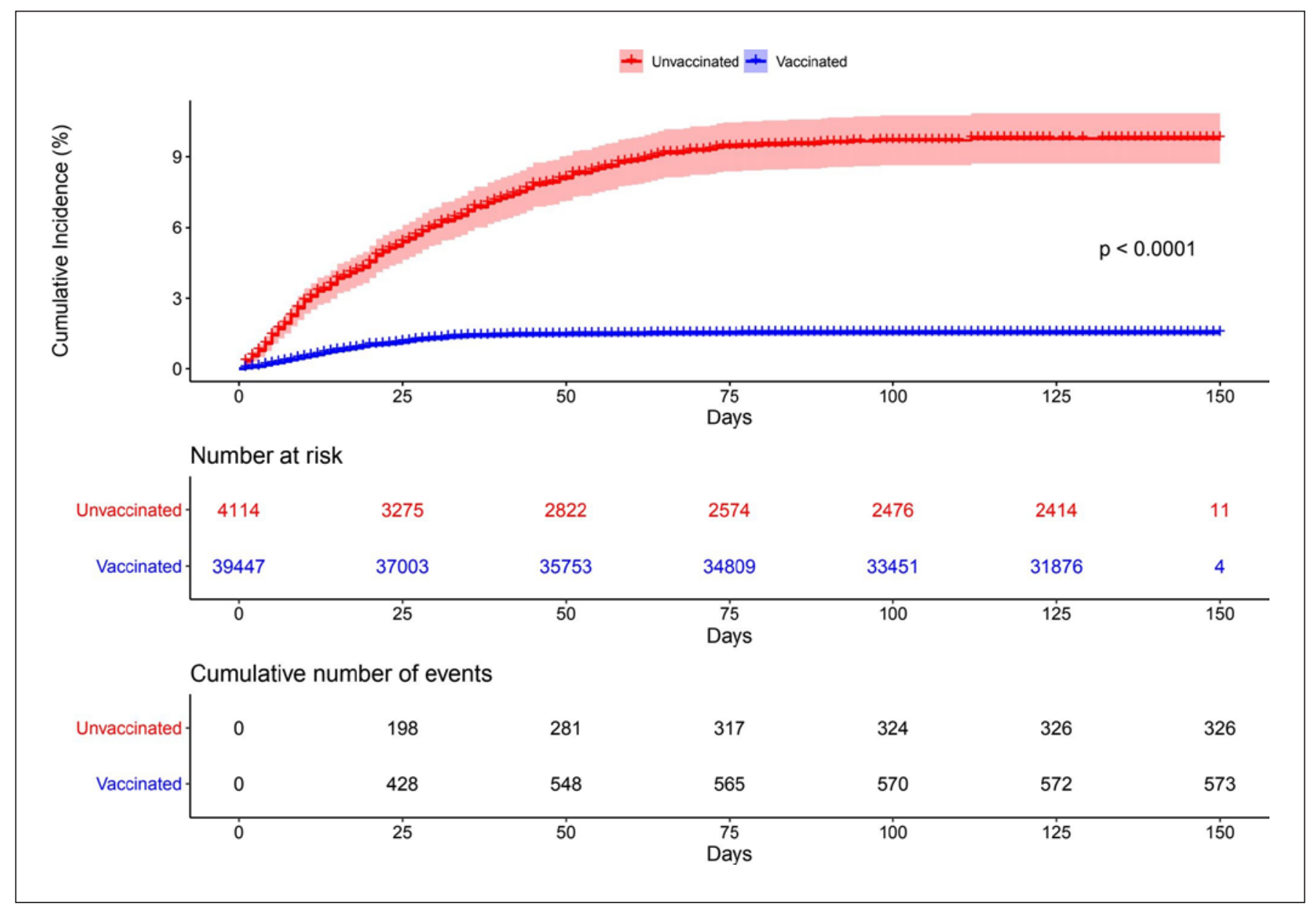

Fig. 2. COVID-19-related death.

\section{Results}

\section{Study Population}

During the study period, there were 92,302 residents in 454 LTCFs in Israel, and 55,744 of them aged 65 years or older. Of those, 43,596 (78.21\%) were eligible for the study (no positive SARS-CoV-2 PCR test or death before the date of the first vaccine dose), with a mean age of 83.0 $( \pm 9.1)$ years.

$90.6 \%(39,482$ residents) of the study population received the first vaccine dose, and $86.3 \%$ (37,656 patients) of the study population received the second vaccine dose. $4.6 \%$ ( 1,826 residents) of the residents who received the first vaccine dose did not receive the second vaccine dose, due to a positive SARS-CoV-2 PCR result (81.3\%, 1,486 residents) and/or death (27.2\%, 497 residents) within 21 days after the receiving the first vaccine dose. These data suggest a mortality rate of $1.25 \%$ in the population of res-

Vaccine Effectiveness in Residents of Long-Term Care Facilities idents that received the first vaccine dose, which is slightly higher than the mortality rate mentioned in the literature for a period of 21 days (1.15\%) [24].

\section{Vaccine Effectiveness}

During the 5-month follow-up period (150 days), a total of 3,742 SARS-CoV-2 infections were documented (0.60 per 1,000 person-days), 899 COVID-related deaths (0.14 per 1,000 person-days), and 3,684 all-cause deaths (0.59 per 1,000 person-days) (Table 1 ). The proportionate mortality from COVID-19 was $21.9 \%$ in the vaccinated population and $30.6 \%$ in the unvaccinated population. The cumulative incidence curves for all outcomes defined are shown in Figures 1-4.

The estimated VE 10 days after the first vaccine dose for SARS-CoV-2 infection was $61.8 \%$ (95\% confidence interval [CI]: 58.2-65.1); COVID-19-related death, 72.3\% (95\% CI: 66.9-76.8); all-cause mortality, 66.9\% (95\% CI: 


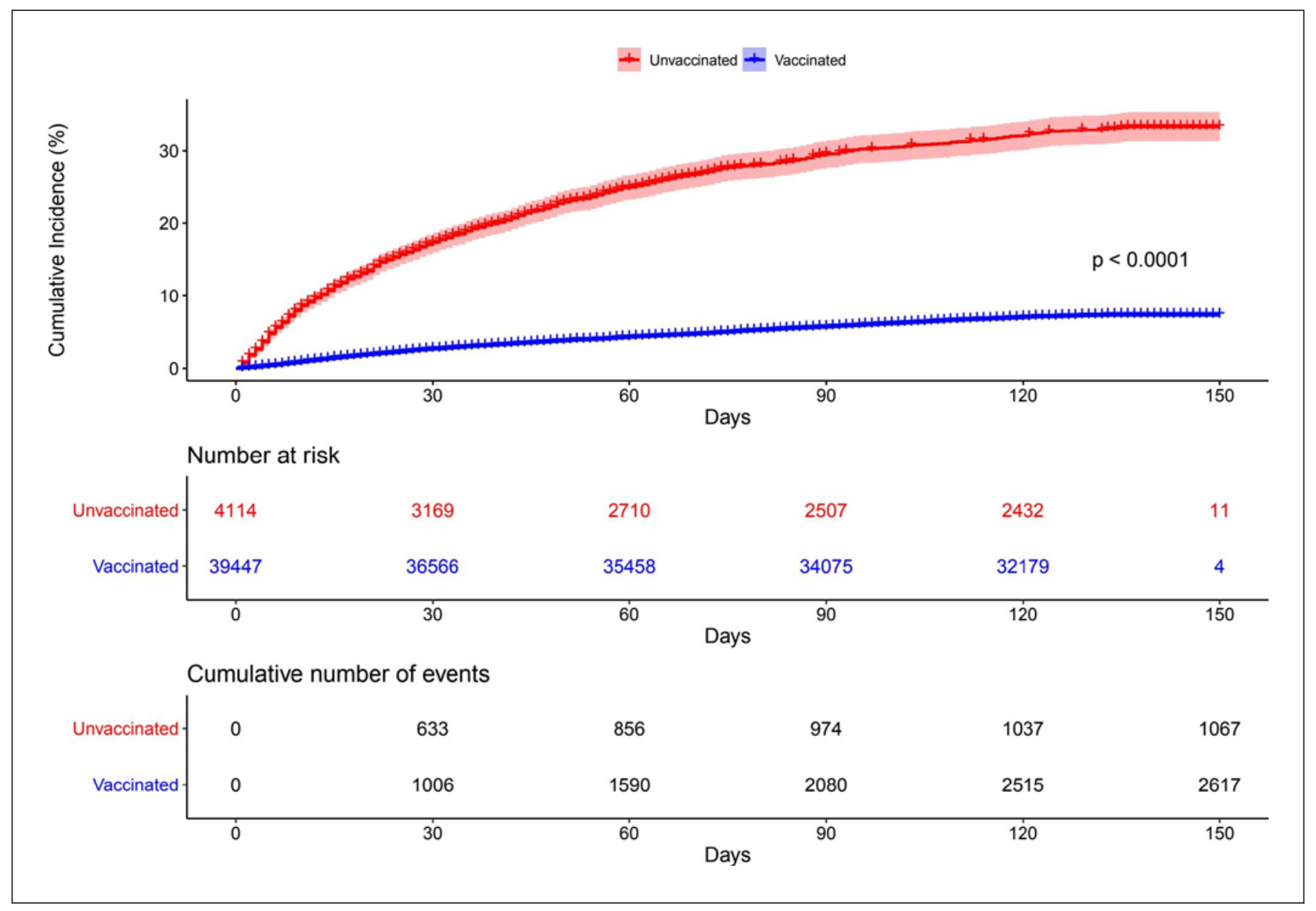

Fig. 3. All-cause mortality.

63.8-69.8); and for the composite endpoint (SARSCoV-2 infection or death), 63.5\% (95\% CI: 60.9-65.9). The estimated VE 28 days after the first vaccine dose, approximately 7 days after the second vaccine dose, for SARS-CoV-2 infection was $81.2 \%$ (95\% CI: 78.6-83.5); COVID related death, $85.3 \%$ (95\% CI: 80.4-88.9); allcause mortality, $63.7 \%$ (95\% CI: 59.4-67.6); and for the composite endpoint (SARS-CoV-2 infection or death), 71.1\% (95\% CI: 68.4-73.5).

\section{The Effect of Age}

VE was affected by age. A 5-year increment in age was associated with decreased vaccine effectiveness; VE decreased in 8.2\% for SARS-CoV-2 infection (95\% CI: 6.110.3), 23.9\% for COVID-19-related death (95\% CI: 19.129.0), 30.1\% for all-cause mortality (95\% CI: 27.5-32.8), and $17.3 \%$ for the composite endpoint (95\% CI: 15.619.1).

\section{Discussion/Conclusion}

\section{Main Results}

This study examined the effectiveness of the BNT162b2 mRNA COVID-19 (Comirnaty) vaccine in a large sample $(43,596)$ of elderly patients living in LTCFs in Israel who received one and two vaccine doses with $90 \%$ and $85.9 \%$ vaccination rates, respectively. Thus, this study is one of the largest cohorts carried out in the elderly population vaccinated with the BNT162b2 mRNA COVID-19 vaccine. Estimated VE during the 5-month follow-up period, 10 days after the first vaccine dose and 7 days after second vaccine dose, was 61.8 and $81.2 \%$ for SARS-CoV-2 infection, 72.3 and $85.3 \%$ for COVID-related death, 66.9 and $63.7 \%$ for all-cause mortality, and 63.5 and $71.1 \%$ for the composite endpoint (SARS-CoV-2 infection or death), respectively. As age increased, the VE decreased. For each 5 -year increment in age, VE decreased in $8.2 \%$ for SARS- 


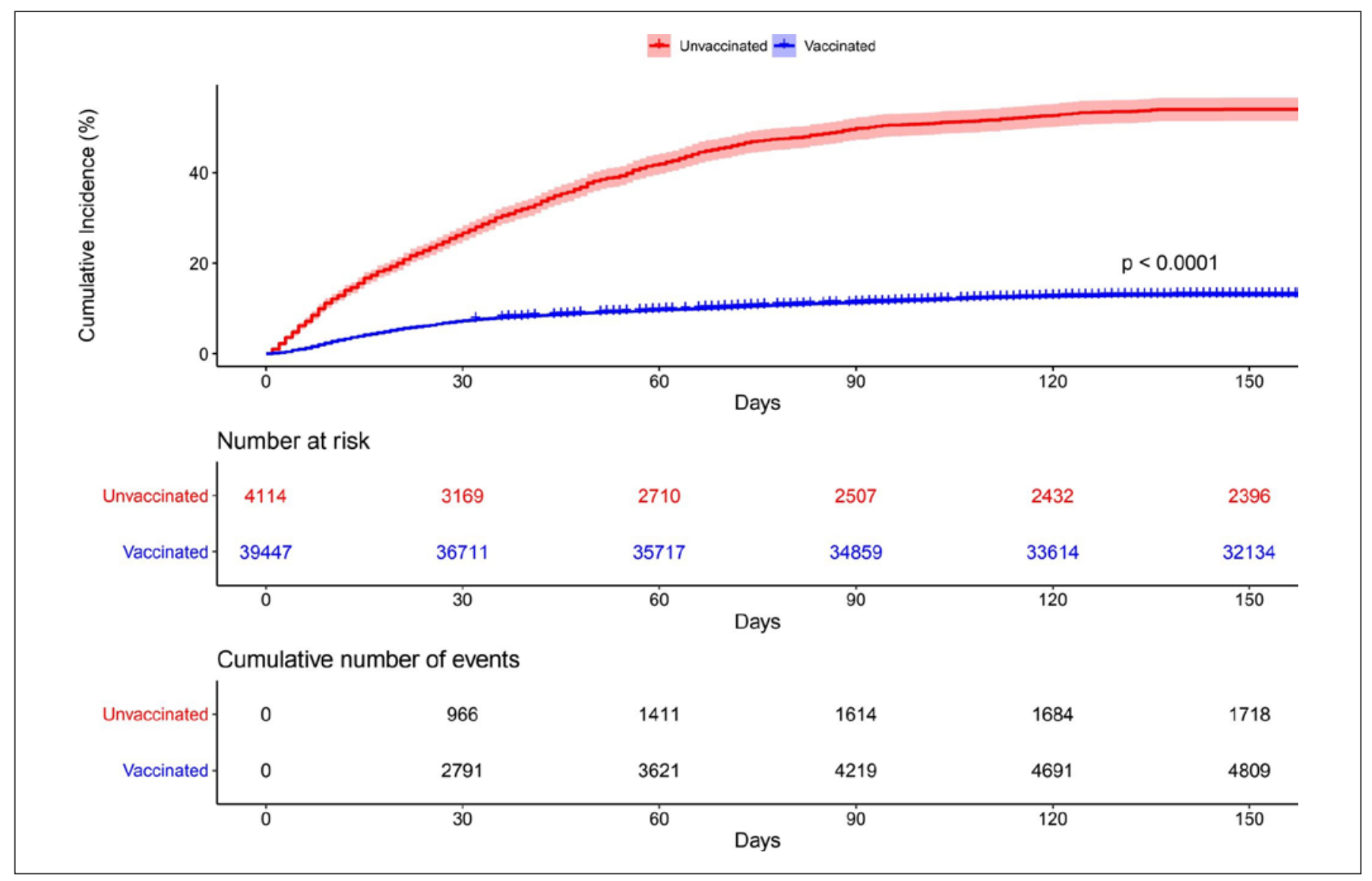

Fig. 4. Composite endpoint - documented SARS-CoV-2 infection or death.

CoV-2 infection, $23.9 \%$ for COVID-19-related death, $30.1 \%$ for all-cause mortality, and $17.3 \%$ for the composite endpoint.

\section{Comparison with the Existing Literature}

Our results align with other studies that showed the BNT162b2 mRNA COVID-19 vaccine effectively reduces the incidence of SARS-Cov-2 infection and COVID-related deaths [25-29]. The effectiveness of the BNT162b2 mRNA COVID-19 vaccine in reducing SARS-CoV-2 infection 28 days after the first dose, approximately 7 days after the second vaccine dose, was $81.2 \%$. This VE was at the high end of VE found in previous studies (56-86.5\%) [19, 22, 28-30]. The effectiveness of the BNT162b2 mRNA COVID-19 vaccine in preventing COVID-related 28 days after the first vaccine dose, approximately 7 days after the second vaccine dose, was $85.3 \%$, lower than the effectiveness as reported by Cavanaugh et al. [30] (94\% >14 days after the second vaccine dose), Mazagatos et al. [31] (97\%), and Starrfelt et al. [32] (93.1\% 7 days after the second vaccine dose).

Vaccine Effectiveness in Residents of Long-Term Care Facilities
The discussion regarding vaccine effectiveness cannot be addressed without attending to nonvaccination and its underlying motives. In the current study, $10 \%$ of the study population was not vaccinated. Previous studies have shown an association between COVID-19 vaccination hesitancy in older population to low income and a low level of education, which is often due to vulnerability to misinformation and personal medical mistrust [33]. In addition, two minority groups in Israel, the ultra-Orthodox Jews and Israeli Arabs, have low immunization rates. This may be related to lack of trust in the government [34]. These factors may cause a selection bias and impact the VE results, as these populations may be more prone to medical illnesses and mortality.

\section{Strengths and Limitations}

The database which was used in this study did not include information about gender or comorbidities of the residents, so we could not examine the effect of these vari- 
ables. However, Mousstsen-Helms et al. [19] showed that adjustments for age, sex, and comorbidities did not affect the VE significantly. Another limitation is a potential selection bias, as we compared a group of vaccinated and unvaccinated residents. The unvaccinated group might have suffered from more comorbidities, leading them to be more susceptible to SARS-CoV-2 infection and death, thus making the vaccine's effectiveness seem higher than it actually is.

The strengths of this study include its nationwide coverage of all residents in all the LTCFs in Israel, its size (43,596 residents), and the stratification by LTCF. In addition, all residents were examined regularly for SARS-CoV-2 infection, so it can be assumed that the vast majority of infections have been identified and documented.

\section{Conclusion}

During the COVID-19 pandemic in Israel, LTCF residents were the most vulnerable population. A lack of official guidelines, a shortage of personal protective equipment, and the poor physical and mental status of residents contributed to this problematic situation. This study shows the effectiveness of the BNT162b2 mRNA COVID-19 vaccine in this population to prevent SARS-2 infection, COVID-19 related death, and all-cause mortality. Further research is warranted on the effect of the third vaccine (booster) in this population.

\section{Statement of Ethics}

This study protocol was reviewed and approved by the Local Ethics Committee of Soroka Medical Center, Ben Gurion University, Beer Sheva, Israel, Approval No. [0429-20-SOR]. Written informed consent was not required, as decided by the Ethics Committee of the Soroka Medical Center.

\section{Conflict of Interest Statement}

During the period described in the study, Sivan Goldin and Nimrod Maimon Held significant positions in the Senior Shield task force. Nimrod Maimon was appointed the head of the task force. Sivan Goldin was responsible for receiving and consolidating information regarding outbreak events in LTCFs across the country and managing severe outbreaks to stop the chains of transmission.

\section{Funding Sources}

No funding was provided to this study.

\section{Author Contributions}

S.G.: conceptualization, methodology, and writing the original draft. L.A.: methodology, formal analysis, visualization, and writing the original draft. J.A.: methodology and writing - reviewing and editing. L.M.: methodology, formal analysis, visualization, and writing - reviewing and editing. S.H.: methodology and writing reviewing and editing. N.M.: conceptualization, supervision, and writing - reviewing and editing.

\section{Data Availability Statement}

Data are not publicly available but can be obtained via reasonable request from the corresponding author.

\section{References}

1 Pandemic experience in the long-term care sector.

2 Centers for medicare \& medicaid services data [Internet]. 2020 [cited 2021 Sep 3]. Available from: https://data.cms.gov/covid-19/covid-19-nursing-home-data.

3 Wang B, Li R, Lu Z, Huang Y. Does comorbidity increase the risk of patients with $\mathrm{CO}$ VID-19: evidence from meta-analysis. Aging. 2020 Apr 8;12:6049-57.

4 Pan AP, Meeks J, Potter T, Masdeu JC, Seshadri S, Smith ML, et al. SARS-CoV-2 susceptibility and COVID-19 mortality among older adults with cognitive impairment: cross-sectional analysis from hospital records in a diverse US metropolitan area. Front Neurol. 2021 Jul 22;12:692662.

5 Niu S, Tian S, Lou J, Kang X, Zhang L, Lian H, et al. Clinical characteristics of older patients infected with COVID-19: a descriptive study. Arch Gerontol Geriatr. 2020 Apr 10;89:104058.
6 Abrams HR, Loomer L, Gandhi A, Grabowski DC. Characteristics of U.S. nursing homes with COVID-19 cases. J Am Geriatr Soc. 2020 Aug;68:1653-6

7 McMichael TM, Currie DW, Clark S, Pogosjans S, Kay M, Schwartz NG, et al. Epidemiology of Covid-19 in a long-term care facility in King County, Washington. N Engl J Med. 2020 May 21;382:2005-11.

8 COVID-19 dashboard report [Internet]. Wayanad: Israeli Ministry of Health; 2021. Available from: https://covidtracer.slemankab.go.id/dashboard2/.

9 European Centre for Disease Prevention and Control. Surveillance of COVID-19 at longterm care facilities in the EU/EEA 2020 May 19. Stockholm: ECDC; 2020.

10 CDC. Interim infection prevention and control recommendations to prevent SARSCoV-2 spread in nursing homes [Internet]. 2021 [cited 2021 Sep 8]. Available from: https://www.cdc.gov/coronavirus/2019ncov/hcp/long-term-care.html?CDC_AA_ refVal=https\%3 A \% 2F\%2 Fwww.cdc. gov\% 2 F c or on avirus \% 2 F 2019 ncov\%2Fhcp\%2Fnursing-homes-responding.html.

11 Ministry of Health. Senior shild [Internet]. 2020. Available from: https://govextra.gov.il/ ministry-of-health/care-covid19/elderlycare-covid19/.

12 Maimon N, Maimon M, Hassan L, Grotto I, Abu-Fraiha Y, Robinson-Geva S, et al. The effect of national weekly COVID-19 screening testing of all workers in long-term care facilities: a decrease in mortality. $2021 \mathrm{Feb}$ 2.

13 Dagan N, Barda N, Kepten E, Miron O, Perchik S, Katz MA, et al. BNT162b2 mRNA Covid-19 vaccine in a nationwide mass vaccination setting. N Engl J Med. 2021 Apr 15;384: 1412-23. 
14 Barda N, Dagan N, Ben-Shlomo Y, Kepten E, Waxman J, Ohana R, et al. Safety of the BNT162b2 mRNA Covid-19 vaccine in a nationwide setting. N Engl J Med. 2021 Aug 25; 385(12): 1078-90.

15 Balicer RD, Ohana R. Israel's COVID-19 endgame. Science. 2021 May 14;372(6543):663.

16 Polack FP, Thomas SJ, Kitchin N, Absalon J, Gurtman A, Lockhart S, et al. Safety and efficacy of the BNT162b2 mRNA Covid-19 vaccine. N Engl J Med. 2020 Dec 31;383:2603-15.

17 Walsh EE, Frenck RW, Falsey AR, Kitchin N, Absalon J, Gurtman A, et al. Safety and immunogenicity of two RNA-based Covid-19 vaccine candidates. N Engl J Med. 2020 Dec 17;383:2439-50.

18 Nace DA, Kip KE, Mellors JW, Peck Palmer OM, Shurin MR, Mulvey K, et al. Antibody responses after mRNA-based COVID-19 vaccination in residential older adults: implications for reopening. J Am Med Dir Assoc. 2021 Aug;22:1593-8.

19 Moustsen-Helms IR, Emborg H-D, Nielsen J, Nielsen KF, Krause TG, Mølbak K, et al. Vaccine effectiveness after 1st and 2nd dose of the BNT162b2 mRNA Covid-19 vaccine in longterm care facility residents and healthcare workers: a Danish Cohort Study. medRxiv [Preprint]. 2021 Mar 9.

20 Torres I, Albert E, Giménez E, Alcaraz MJ, Botija P, Amat P, et al. B- and T-cell immune responses elicited by the Comirnaty ${ }^{\oplus} \mathrm{CO}$ VID-19 vaccine in nursing-home residents. Clin Microbiol Infect. 2021 Jun 24;27(11): $1672-7$.

21 Canaday DH, Carias L, Oyebanji OA, Keresztesy D, Wilk D, Payne M, et al. Reduced BNT162b2 mRNA vaccine response in SARS$\mathrm{CoV}$-2-naive nursing home residents. Clin Infect Dis. 2021 May 16;73(11):2112-5.
22 State Bar. Fictitiousness under cover of the state [Internet]. Calcalist. 2018 Feb 25 [cited 2021 Dec 4]. Available from: https://www.calcalist.co.il/local/articles/0.7340.L-3732625.00. html.

23 Completing birth date procedure [Internet]. 2014 Oct 1 [cited 2021 Dec 5]. Available from: https: //www.gov.il/BlobFolder/policy/completing_birth_date_procedure/he/2.7.0002. pdf.

24 Brink P, Kelley ML. Death in long-term care: a brief report examining factors associated with death within 31 days of assessment. Palliat Care. 2015 Feb 1;9:1-5.

25 White EM, Yang X, Blackman C, Feifer RA, Gravenstein S, Mor V. Incident SARS-CoV-2 infection among mRNA-vaccinated and unvaccinated nursing home residents. $\mathrm{N}$ Engl J Med. 2021 Jul 29;385(5):474-6.

26 Salcher-Konrad M, Smith S, Comas-Herrera A. Emerging evidence on effectiveness of COVID-19 vaccines among residents of longterm care facilities. J Am Med Dir Assoc. 2021 Aug;22(8):1602-3.

27 Mor V, Gutman R, Yang X, White EM, McConeghy KW, Feifer RA, et al. Short-term impact of nursing home SARS-CoV-2 vaccinations on new infections, hospitalizations, and deaths. J Am Geriatr Soc. 2021 Apr 16;69(8): 2063-9.

28 De Salazar PM, Link N, Lamarca K, Santillana M. High coverage COVID-19 mRNA vaccination rapidly controls SARS-CoV-2 transmission in long-term care facilities. Res Sq [Preprint]. 2021 Apr 12.
29 Domi M, Leitson M, Gifford D, Nicolaou A, Sreenivas K, Bishnoi C. The BNT162b2 vaccine is associated with lower new COVID-19 cases in nursing home residents and staff. J Am Geriatr Soc. 2021 Aug;69(8):2079-89.

30 Cavanaugh AM, Fortier S, Lewis P, Arora V, Johnson M, George K, et al. COVID-19 outbreak associated with a SARS-CoV-2 R.1 lineage variant in a skilled nursing facility after vaccination program: Kentucky, March 2021. MMWR Morb Mortal Wkly Rep. 2021 Apr 30;70:639-43.

31 Mazagatos C, Monge S, Olmedo C, Vega L, Gallego P, Martín-Merino E, et al. Effectiveness of mRNA COVID-19 vaccines in preventing SARS-CoV-2 infections and COVID-19 hospitalisations and deaths in elderly long-term care facility residents, Spain, weeks 532020 to 132021 . Euro Surveill. 2021 Jun;26: 2100452.

32 Starrfelt J, Danielsen AS, Kacelnik O, Børseth AW, Seppälä E, Meijerink H. High vaccine effectiveness against COVID-19 infection and severe disease among residents and staff of long-term care facilities in Norway, November-June 2021. medRxiv [Preprint]. 2021 Aug 9.

33 Veronese N, Saccaro C, Demurtas J, Smith L, Dominguez LJ, Maggi S, et al. Prevalence of unwillingness and uncertainty to vaccinate against COVID-19 in older people: a systematic review and meta-analysis. Ageing Res Rev. 2021 Dec;72:101489.

34 Rosen B, Waitzberg R, Israeli A, Hartal M, Davidovitch N. Addressing vaccine hesitancy and access barriers to achieve persistent progress in Israel's COVID-19 vaccination program. Isr J Health Policy Res. 2021 Aug 2; 10(1):43. 